\title{
EFEITO DE AGROTÓXICOS NA PATOGENICIDADE DOS CONÍDIOS DE LECANICILLIUM LECANII A TETRANYCHUS URTICAE
}

\section{I.M. Wenzel; A. Batista Filho}

Instituto Biológico, Centro Experimental Central, CP 70, CEP 13001-970, Campinas, SP, Brasil. E-mail: iawenzel@yahoo.com.br

\section{RESUMO}

\begin{abstract}
O objetivo do trabalho foi verificar a patogenicidade dos conídios do isolado JAB 02 de Lecanicillium lecanii, produzidos em meio de cultura contendo acaricidas e inseticidas, ao ácaro rajado Tetranychus urticae. Foi feita uma mistura dos inseticidas (acefato, ciromazina, endosulfan, cloridrato de formetanato, imidacloprido, metiocarbe, metomil, monocrotofós e tiametoxam) e acaricidas (abamectina e propargite) ao meio de cultura BDA que foi vertido em placas de Petri. Após a solidificação, a inoculação do fungo foi realizada com alça de platina, através de três pontos, e as placas foram incubadas durante 16 dias em temperatura de $25,5 \pm 0,5^{\circ} \mathrm{C}$ e fotofase de 12 horas. Após o crescimento do fungo, as colônias foram recortadas e transferidas para tubos de ensaio contendo água destilada e espalhante adesivo. A suspensão obtida de cada tratamento foi padronizada em $1 \times 10^{7} \mathrm{con}$./ $\mathrm{mL}$ e $1 \mathrm{~mL}$ foi pulverizado em folhas de feijão de porco. Posteriormente à pulverização, os ácaros foram transferidos para as folhas e a mortalidade avaliada, sob microscópio estereoscópico, no $2^{\circ}, 4^{\circ}$ e $6^{\circ}$ dias após a aplicação. A presença dos acaricidas e inseticidas no meio de cultivo não afetou a patogenicidade dos conídios de L. lecanii que causaram no $6^{\circ}$ dia de avaliação mortalidades confirmadas de 47 a $76 \%$ do ácaro rajado T. urticae.
\end{abstract}

PALAVRAS-CHAVE: Fungo entomopatogênico, seletividade, ácaro rajado, controle microbiano, compatibilidade.

\section{ABSTRACT}

PESTICIDE EFFECTS ON THE PATHOGENICITY OF CONIDIA OF LECANICILLIUM LECANII TO TETRANYCHUS URTICAE. The objective of this study was to evaluate the effect of pesticides on the pathogenicity of conidia of Lecanicillium lecanii, strain JAB 02, produced in culture media containing acaricides and insecticides, to the two-spotted mite Tetranychus urticae. A blend was made of insecticides (acephate, cyromazine, endosulfan, formetanate hydrochloride, imidacloprid, methiocarb, methomyl, monocrotophos, thiamethoxam) and acaricides (abamectin and propargite) in the culture media PDA, that was poured into a Petri dishes. After solidification, the fungus inoculation was realized at three points and the dishes were incubated for 16 days at a temperature of $25.5 \pm 0.5^{\circ} \mathrm{C}$ and 12 hours of photophase. After the fungus growth, the colonies were cut out and removed to test tubes containing distilled water and detergent Tween $80^{\circ}$. The fungus suspension obtained for each treatment was standardized at $1 \times 10^{7} \mathrm{con} . / \mathrm{mL}$ and $1 \mathrm{~mL}$ was sprayed on Canavalia ensiformis leaves. After the spraying, 15 mites were placed on each leaf disk and after 2, 4 and 6 days the mortality was evaluated in a stereoscopic microscope. The presence of acaricides and insecticides in the culture media did not affect the pathogenicity of conidia of $L$. lecanii, which on the 6th evaluation day presented a confirmed mortality in the range of 47 to $76 \%$ on two-spotted mites T. urticae.

KEYWORDS: Entomopathogenic fungus, selectivity, two-spotted mite, microbial control,compatibility.

\section{INTRODUÇÃO}

A patogenicidade é a capacidade de um microorganismo causar uma doença. A mortalidade causada por fungos em artrópodos geralmente está relacionada a vários fatores como deficiência nutricional, invasão e destruição dos tecidos eliberação de toxinas no hospedeiro (TANADA; KAYA, 1992). Avaliar a patogenicidade de fungos a insetos e ácaros é de extrema importância, pois permite que seja feita uma seleção de espécies e isolados mais virulentos à praga-alvo e adequados ao seu hábito e sua biologia. Os fungos entomopatogênicos podem causar epizootias naturais, infectando diferentes estágios de desenvolvimento dos hospedeiros como ovo, larva, pupa e adulto (ALVES; LECUONA, 1998). Alguns fatores 
abióticos como temperatura e umidade relativa podem afetar a habilidade e velocidade com a qual os fungos podem infectar e colonizar o inseto hospedeiro (Yeo et al., 2003). Segundo HelYer et al. (1992), o fungo Lecanicillium lecanii para ser efetivo requer condições ambientais adequadas como temperatura entre 15 e $25^{\circ} \mathrm{C}$ e umidade relativa alta.

Outros fatores, como o uso indiscriminado de agrotóxicos, também podem afetar diretamente a capacidade patogênica dos fungos. Aplicações regulares de um amplo espectro de agroquímicos, incluindo agrotóxicos, podem resultar na sua acumulação no meio ambiente e, consequentemente, alterar componentes biológicos (POPRAWSKI; MAJCHROWICZ, 1995).

No crisântemo, uma das principais pragas é o ácaro rajado Tetranychus urticae, uma espécie cosmopolita e polífaga de grande importância econômica (Draganova; Simova, 2010), cujo controle é quase que exclusivamente realizado por meio da aplicação de acaricidas que pode desencadear efeitos indesejáveis como mortalidade de organismos não alvos, desenvolvimento de resistência, entre outros (SKIRVIN; Williams, 1999).

Agentes de biocontrole se mostram como uma alternativa aos controles tradicionais (DRAGANOVA; SIMOVA, 2010). Os fungos patogênicos Beauveria bassiana, Metarhiziumanisopliae, Isaria fumosorosea, Isariafarinosa, Hirsutella thompsonii, Neozygites floridana e L. lecanii exercem papel importante na diminuição das populações nativas de ácaros fitófagos (CHANDLER et al., 2000, VAN DER GEEST et al., 2000).

WeNZEL (2005) verificou que L. lecanii foi patogênico ao ácaro rajado causando índices de mortalidade variando entre 49 e $90 \%$, para concentrações variando entre $5 \times 10^{6}$ a $5 \times 10^{8}$ conídios $/ \mathrm{mL}$, e mostrando uma possível alternativa no controle desta praga.

Estudos de compatibilidade, em laboratório, usualmente têm como parâmetros o crescimento vegetativo e a esporulação do fungo, não levando em consideração a capacidade do fungo em continuar infectivo. Devido à importância do assunto e a escassez de publicações relacionadas ao tema, o presente trabalho teve como objetivo avaliar a influência de agrotóxicos utilizados no cultivo do crisântemo, sobre a patogenicidade de conídios de L. lecanii a T. urticae.

\section{MATERIAL E MÉTODOS}

\section{Micro-organismo utilizado}

Foi utilizado o isolado JAB 02 de Lecanicillium lecanii depositado no Laboratório de Ecologia de Micro-organismos da Faculdade deCiências Agrárias e Veterinárias/Universidade Estadual Paulista,
Jaboticabal, SP, Brasil, proveniente da cochonilha verde Coccus viridis Green (Hemiptera: Coccidae). $\mathrm{O}$ fungo, conservado em óleo mineral, foi multiplicado por meio de repicagens em placas de Petri com BDA. O período de incubação foi de 12 dias a $25,5 \pm 0,5^{\circ} \mathrm{C}$ em B.O.D., para obtenção de culturas jovens com vistas à utilização nos ensaios.

Patogenicidade de L. lecanii, cultivado em meio de cultura contendo inseticidas e acaricidas, ao ácaro rajado $T$. urticae

O fungo L. lecanii foi repicado em meio de cultura BDA (batata-dextrose-ágar) contendo os agrotóxicos na dosagem estipulada pelofabricante (para produtos cujo uso é recomendado no cultivo do crisântemo) e na dosagem utilizada pelos produtores (para produtos que não são recomendados para este cultivo, mas que são utilizados pelos produtores) misturados proporcionalmente à quantidade de $200 \mathrm{~mL}$ de meio de cultura (Tabela 1). O meio de cultura foi autoclavado a $1 \mathrm{~atm}\left(121^{\circ} \mathrm{C}\right)$ por 20 minutos e, antes da solidificação, a uma temperatura deaproximadamente $45^{\circ} \mathrm{C}$ foram acrescentados os produtos nas devidas proporções. Em seguida, a mistura foi vertida em placas de Petri de $9 \mathrm{~cm}$ de diâmetro e a testemunha fúngica foi representada pelo meio de cultura sem produto. Após a solidificação do meio, o fungo foi transferido através de três pontos equidistantes nas placas, com auxílio de uma alça de platina. As placas repicadas foram mantidas em câmara de germinação (B.O.D.) durante 16 dias à temperatura de $25,5 \pm 0,5^{\circ} \mathrm{C}$ e fotofase de 12 horas. Após este tempo, as colônias foram recortadas com estilete flambado e colocadas em tubos de ensaio com $10 \mathrm{~mL}$ de uma suspensão autoclavada de água destilada e espalhante adesivo (Tween $80^{\circledR}$ ) a $0,1 \%$. Essa suspensão foi agitada em agitador elétrico de tubos, para extração e posterior contagem dos conídios em câmara de Neubauer para padronização das suspensões.

Para o preparo da viabilidade, retirou-se uma alíquota de 0,1 mL, desta mesma suspensão, e espalhou-se com auxílio de uma alça de Drigalsky previamenteflambada edevidamenteresfriada, numa placa de Petri de plástico contendo uma fina camada de BDA com pentabiótico $(0,5 \mathrm{~g} / \mathrm{L})$. Após 15 horas de incubação à temperatura de $25,5 \pm 0,5^{\circ} \mathrm{C}$ e fotofase de 12 horas, foram observados em microscópio óptico, em aumento de 400 x, os conídios germinados e não germinados, estabelecendo-se uma proporção.

Para o teste de patogenicidade foi utilizado oácaro rajado T. urticae criado no Laboratório de Controle Biológico do Instituto Biológico, Campinas, SP, em folhas de feijão de porco (Canavalia ensiformis L.). As suspensões obtidas dos tratamentos com cada produto e da testemunha fúngica foram padronizadas em $1 \times 10^{7}$ conídios/ $\mathrm{mL}$ e $1 \mathrm{~mL}$ foi pulverizado 
por meio da Torre de Potter, na pressão de $15 \mathrm{lb} /$ $\mathrm{pol}^{2}$, nas arenas montadas em placas de Petri de $9 \mathrm{~cm}$ de diâmetro, com discos de folhas de feijão recortadas com o mesmo diâmetro e algodão umedecido em toda a lateral da placa, servindo como uma barreira com a finalidade de evitar a fuga dos ácaros. Para a testemunha absoluta foi pulverizado $1 \mathrm{~mL}$ de água destilada.

Após uma breve secagem da suspensão, cada placa recebeu 15 ácaros/disco de folha, sendo posteriormente acondicionadas em BOD a $25,5 \pm 0,5^{\circ} \mathrm{C}$. A avaliação ocorreu com auxílio de um microscópio estereoscópico aos 2, 4 e 6 dias após a aplicação do fungo. Com auxílio de uma alça de platina, os ácaros mortos foram cuidadosamente transferidos para câmaras úmidas confeccionadas com placas de Petri de $9 \mathrm{~cm}$ de diâmetro contendo um pedaço de algodão umedecido e seladas com filme plástico, para a confirmação da morte pelo entomopatógeno.

Foram realizados cinco ensaios distintos devido ao número de produtos testados etambém ao número de ácaros disponível na criação.

\section{Análise estatística}

Odelineamento experimental de todos os ensaios foi inteiramente casualizado (DIC) com 6 repetições e a análise foi realizada através do programa ESTAT 2.0. Os dados foram transformados em $\sqrt{x+10}$ e submetidos à análise de variância. Na significância desta, foi feito o teste de Tukey a $5 \%$ para a comparação entre as médias.

\section{RESULTADOS E DISCUSSÃO}

A viabilidade média dos conídios verificada na testemunha fúngica e nos demais tratamentos com agrotóxicos foi maior que $92 \%$.
Não foi observada mortalidade confirmada na testemunha absoluta (apenas aplicação de água) mostrando que não houve contaminação do tratamento. A mortalidade na testemunha absoluta e as mortalidades não confirmadas nos demais tratamentos ficaram abaixo de $20 \%$ durante todo o período de avaliação do ensaio.

No ensaio 1, não houve diferença estatística quanto à mortalidade após 2, 4 e 6 dias da aplicação para os tratamentos com imidacloprido na formulação GRDA (doses máxima e mínima), acefato e JAB 02. No sexto dia de avaliação, as mortalidades confirmadas variaram entre 57 e 65,5\% (Tabela 2). No ensaio 2, no segundo dia de avaliação a maior porcentagem de mortalidade foi observada para o tratamento com abamectina (dose mínima), queapresentou diferença estatística em relação aos demais tratamentos. No quarto dia, as mortalidades de $T$. urticae foram estatisticamente iguais para abamectina (dose mínima), endosulfan (dose mínima) e o tratamento JAB 02. No sexto dia não houve diferença estatística entre os tratamentos e as mortalidades no final do período de avaliação variaram entre 52 e $67 \%$ (Tabela 2).

A testemunha fúngica, no ensaio 3 , diferiu estatisticamente do metiocarbe no quarto dia de avaliação. Os tratamentos JAB 02, tiametoxam e cloridrato de formetanato foram estatisticamente iguais entre si nas três avaliações. No sexto dia o tratamento JAB 02 apresentou mortalidade de $76 \%$ (Tabela 2).

Não houve diferença estatística, no ensaio 4 , entre os tratamentos JAB 02 , monocrotofós e metomil, com mortalidades variando entre 47 a 56\% (Tabela 2). O mesmo foi observado no ensaio 5, para JAB 02, propargito, imidacloprido (formulação SC) e ciromazina com mortalidades confirmadas variando de 53 a $70 \%$ no sexto dia de avaliação.

Tabela 1 - Inseticidas e acaricidas recomendados e utilizados para controle de pragas da cultura do crisântemo(AGROFIT, 2010). * Quantidades adicionadas em $200 \mathrm{~mL}$ de meio de cultura.

\begin{tabular}{|c|c|c|c|}
\hline Ingrediente ativo & Produto comercial & Dose de i.a.* & Dose do p.c.* \\
\hline & Inseticidas & & \\
\hline tiametoxam & Actara $250 \mathrm{WG}$ & $0,2 \mathrm{~g}$ & $0,8 \mathrm{~g}$ \\
\hline monocrotofós & Azodrin 400 & $0,08 \mathrm{~g}$ & $0,2 \mathrm{~mL}$ \\
\hline imidacloprido & Confidor 700 GRDA & 0,14 e $0,504 \mathrm{~g}$ & 0,2 e $0,72 \mathrm{~g}$ \\
\hline cloridrato de formetanato & Dicarzol 500 PS & $0,1746 \mathrm{~g}$ & $0,3 \mathrm{~g}$ \\
\hline metomil & Lannate BR & $0,043 \mathrm{~g}$ & $0,2 \mathrm{~mL}$ \\
\hline metiocarbe & Mesurol 500 SC & $0,15 \mathrm{~g}$ & $0,3 \mathrm{~mL}$ \\
\hline acefato & Orthene 750 BR & $0,15 \mathrm{~g}$ & $0,2 \mathrm{~g}$ \\
\hline imidacloprido & Provado 200 SC & $0,04 \mathrm{~g}$ & $0,2 \mathrm{~mL}$ \\
\hline endosulfan & Thiodan EC & 0,7 e $1,05 \mathrm{~g}$ & 2,0 e $3,0 \mathrm{~mL}$ \\
\hline \multirow[t]{2}{*}{ ciromazina } & Trigard 750 PM & $0,0225 \mathrm{~g}$ & $0,03 \mathrm{~g}$ \\
\hline & Acaricidas & & \\
\hline propargito & Omite 720 EC BR & $0,432 \mathrm{~g}$ & $0,06 \mathrm{~mL}$ \\
\hline abamectina & Vertimec $18 \mathrm{CE}$ & 0,018 e $0,036 \mathrm{~g}$ & 0,05 e $0,1 \mathrm{~mL}$ \\
\hline
\end{tabular}


Tabela 2 - Mortalidade confirmada (\%) de Tetranychus urticae após pulverização de conídios do isolado JAB 02 de Lecanicillium lecanii produzidos em meio de cultura contendo inseticidas e acaricidas $\left(\mathrm{T}=25,5 \pm 0,5^{\circ} \mathrm{C}\right.$ e fotofase de 12 horas).

\begin{tabular}{|c|c|c|c|}
\hline Tratamentos & $2 \operatorname{dias}^{1}$ & $4 \operatorname{dias}^{1}$ & 6 dias $^{1}$ \\
\hline JAB 02 & $7,0 \mathrm{a}$ & $33,0 \mathrm{a}$ & $57,5 \mathrm{a}$ \\
\hline Imidacloprido GRDA máx. & $12,0 \mathrm{a}$ & $43,0 \mathrm{a}$ & $62,0 \mathrm{a}$ \\
\hline Imidacloprido GRDA mín máx mín. & $13,0 \mathrm{a}$ & $44,0 \mathrm{a}$ & $65,5 \mathrm{a}$ \\
\hline Acefato & $20,0 \mathrm{a}$ & $44,0 \mathrm{a}$ & $64,0 \mathrm{a}$ \\
\hline Teste F & $1,01^{\text {n.s. }}$ & $0,98^{\text {n.s. }}$ & $0,40^{\text {n.s. }}$ \\
\hline C.V. $(\%)$ & 24,15 & 12,96 & 10,14 \\
\hline Tratamentos & 2 dias & 4 dias & 6 dias \\
\hline JAB 02 & $4,5 \mathrm{~b}$ & $28,0 \mathrm{ab}$ & $52,0 \mathrm{a}$ \\
\hline Endosulfan mín. & $10,0 \mathrm{~b}$ & $35,0 \mathrm{ab}$ & $67,0 \mathrm{a}$ \\
\hline Abamectina máx. & $7,0 \mathrm{~b}$ & $26,0 \quad b$ & $55,0 \mathrm{a}$ \\
\hline Abamectina mín. & $29,0 \mathrm{~b}$ & $49,0 \mathrm{a}$ & $61,0 \mathrm{a}$ \\
\hline Teste F & $11,05^{\star *}$ & $3,88^{*}$ & $1,16^{\text {n.s. }}$ \\
\hline C.V. $(\%)$ & 17,85 & 14,56 & 12,09 \\
\hline Tratamentos & 2 dias & 4 dias & 6 dias \\
\hline JAB 02 & $2,5 a$ & $51,0 \mathrm{a}$ & $76,0 \mathrm{a}$ \\
\hline Tiametoxam & $2,0 \mathrm{a}$ & $33,0 \mathrm{ab}$ & $70,0 \mathrm{a}$ \\
\hline Cloridrato de formetanato & $4,0 \mathrm{a}$ & $28,5 \mathrm{ab}$ & $61,0 \mathrm{a}$ \\
\hline Metocarbe & $1,5 \mathrm{a}$ & $22,0 \mathrm{~b}$ & $50,0 \mathrm{a}$ \\
\hline Teste F & $0,49^{\text {n.s. }}$ & $3,09^{*}$ & $2,74^{\text {n.s. }}$ \\
\hline C.V. $(\%)$ & 16,56 & 20,06 & 12,16 \\
\hline Tratamentos & 2 dias & 4 dias & 6 dias \\
\hline JAB 02 & $2,0 \mathrm{a}$ & $35,0 \mathrm{a}$ & $56,0 \mathrm{a}$ \\
\hline Monocrotofós & $5,0 \mathrm{a}$ & $25,0 \mathrm{a}$ & 47,0 a \\
\hline Metomil & $9,0 \mathrm{a}$ & $25,0 \mathrm{a}$ & $48,0 \mathrm{a}$ \\
\hline Teste F & $1,59^{\text {n.s. }}$ & $1,91^{\text {n.s. }}$ & $0,84^{\text {n.s. }}$ \\
\hline C.V. $(\%)$ & 20,23 & 14,30 & 12,15 \\
\hline Tratamentos & 2 dias & 4 dias & 6 dias \\
\hline JAB 02 & $10,5 \mathrm{a}$ & $25,0 \mathrm{a}$ & $53,0 \mathrm{a}$ \\
\hline Propargito & $5,0 \mathrm{a}$ & $35,0 \mathrm{a}$ & $65,0 \mathrm{a}$ \\
\hline Imidacloprido SC & $6,0 \mathrm{a}$ & $32,0 \mathrm{a}$ & $59,0 \mathrm{a}$ \\
\hline Ciromazina & $14,0 \mathrm{a}$ & $38,5 \mathrm{a}$ & $70,0 \mathrm{a}$ \\
\hline Teste F & $1,17^{\text {n.s. }}$ & $1,36^{\text {n.s. }}$ & $1,88^{\text {n.s. }}$ \\
\hline C.V. $(\%)$ & 25,07 & 15,99 & 9,45 \\
\hline
\end{tabular}

${ }^{1}$ Dados originais na tabela, porém transformados em $\sqrt{ } \mathrm{x}=10$ para análise estatística.

Médias seguidas de mesma letra na coluna não diferem entre si pelo teste de Tukey a 5\% de probabilidade.

** significativo a $1 \%$ de probabilidade.

n.s. $=$ não significativo.

C.V. = coeficiente de variação.

WeNZEL et al., (2004) previamente testaram a patogenicidade dos conídios de L. lecanii, produzidos em meio de cultivo contendo dois inseticidas. Os autores verificaram que não houve influência dos produtos sobre o fungo, pois, as mortalidades confirmadas de T. urticae foram de $81 \%$ para imidacloprido e $85 \%$ para ciromazina.

A patogenicidade, em lagartas de Galleriamellonella L., deconídios dofungo Beauveria bassiana (Bals) Vuill. produzidos em meios de cultura com os produtos tiametoxam, imidacloprido, iprodioneefenpropatina foi estudada por CAVALCANTI et al. (2002). Os autores observaram resultados semelhantes aos obtidos, pois não verificaram diferença significativa na mortalidade de G. mellonella para os tratamentos contendo agrotóxicos e a testemunha. No entanto, observaram que para o produto iprodione a porcentagem de mortalidade foi de $48 \%$ enquanto na testemunha foi de $84 \%$, sugerindo que pode ter havido alguma ação prejudicial do produto sobre o fungo.

GASSEN etal. (2008) também avaliaram a patogenicidade de conídios de B. bassiana em lagartas de G. 
mellonella após cultivar o fungo em meio contendo agrotóxicos e verificaram que não houve diferença estatística entre a mortalidade na testemunha e no tratamento com tiametoxam concordando com os resultados obtidos neste trabalho.

A presença dos agrotóxicos no meio de cultura não foi suficiente para causar uma ação deletéria nos conídios, pois os sintomas apresentados pelos ácaros foram típicos de hospedeiros infectados por fungos como tremores, debilidade e/ ou dificuldade de locomoção e extrusão do fungo dando a coloração esbranquiçada e aspecto algodonoso ao ácaro (TAKADA, 2002), mostrando que os agrotóxicos não influenciaram a capacidade do conídio causar a doença e completar seu ciclo no hospedeiro. A germinação do conídio, uma das etapas consideradas mais importantes do ciclo, não foi afetada pelos produtos químicos. A germinação é dependente das condições nutricionais e a ausência, qualitativa e quantitativa de nutrientes, afeta a germinação, assim como componentes do tegumento do hospedeiro entre outros (TANADA; KAYA, 1992).

A efetividade dos inseticidas microbianos depende de vários fatores incluindo a suscetibilidade natural da praga-alvo ao grau de exposição ao produto microbiano, às condições ambientais e às interações fisiológicas entre hospedeiro e patógeno. Geralmente, os primeiros instares são mais suscetíveis aos patógenos. Insetos sobre "stress", assim como aqueles enfraquecidos por outro patógeno ou por condição alimentar inadequada ou ainda agrupados, são mais facilmente controlados do que os insetos em condições normais (TANADA; KAYA, 1992).

A interação entre produtos fitossanitários e entomopatógenos deve ser considerada antes da recomendação do agente químico e representa uma importante ferramenta nos programas de MIP (BATISTA FILHO et al., 2001)

\section{CONCLUSÕES}

Os inseticidas e acaricidas misturados ao meio de cultura não afetam a capacidade infectiva dos conídios L. lecanii ao ácaro T. urticae.

Os inseticidas tiametoxam, monocrotofós, imidacloprido, cloridrato de formetanato, metomil, metiocarbe, acefato, endosulfan, ciromazina e os acaricidas abamectina e propargito utilizados neste trabalho podem ser selecionados para uso no Manejo Integrado de Pragas.

\section{REFERÊNCIAS}

AGROFIT, 2010. Registro de agrotóxicos e afins constantes. Disponível em: <http:/ /agrofit.agricultura.gov. br/agrofit_cons/principal_agrofit_cons>. Acesso em: 21 abr 2009.

ALVES, S.B.; LECUONA, R.G. Epizootiologia aplicada ao controle microbiano de insetos. In: ALVES, S.B. (Ed.). Controle microbiano de insetos. Piracicaba: FEALQ, 1998. p.97-169.

BATISTA FILHO, A.; ALMEIDA, J.E.M.; LAMAS, C. Effect of thiamethoxam on entomopathogenic microorganisms. Neotropical Entomology, v.30, n.3, p.437-447, 2001.

CAVALCANTI, R.S.; MOINO JUNIOR, A.; SOUZA, G.C.; ARNOSTI, A. Efeito de produtos fitossanitários fenpropatrina, imidaclopride, iprodione, e tiametoxam sobre o desenvolvimento do fungo Beauveria bassiana (Bals.) Vuill. Arquivos do Instituto Biológico, São Paulo, v.69, n.3, p.17-22, 2002.

CHANDLER, D.; DAVIDSON, G.; PELL, J. K.; BALL,B . V.; SHAW, K.; SUNDERLAND, K. D. Fungal control of Acari. Biocontrol Science and Technology, v.10, n.4, p.357384, 2000.

DRAGANOVA, S.A.; SIMOVA, S.A. Susceptibility of Tetranychus urticae Koch. (Acari: Tetranychidae) to isolates of entomopathogenic fungus Beauveria bassiana. Pesticides and Phytomedicine, v.25, n.1, p.51-57, 2010.

GASSEN, M.H.; BATISTA FILHO, A.; ZAPPELINI, L.O.; WENZEL, I.M. Efeito de agrotóxicos utilizados na cultura da goiaba sobre o fungo entomopatogênico Beauveria bassiana (Balls.) Vuill. Arquivos do Instituto Biológico, São Paulo, v.75, n.3, p.327-342, 2008.

HELYER, N.; GILL, G.; BYWATER, A.; CHAMBERS, R. Elevated humidities for control of chrysanthemum pests with Verticillium lecanii. Pesticide Science, v.36, p.373-378, 1992.

POPRAWSKI, T.J.; MAJCHROWICZ, I. Effects of herbicides on in vitro vegetative growth and sporulation of entomopathogenic fungi. Crop Protection, v.14, n.1, p.81-87, 1995.

SKIRVIN, D. J.; WILLIAMS, M. C. Differential effects of plant species on a mite pest (Tetranychus urticae) and its predator (Phytoseiulus persimilis): implications for biological control. Experimental and Applied Acarology, v.23, p.497-512, 1999.

TAKADA, H.M. Seleção de isolados de Metarhizium anisopliae (Metsch.) Sorokin e Beauveria bassiana (Bals.) Vuil. para controle de Oryzophagus oryzae (Costa Lima, 136) (Coleoptera: Curculionidae). 2002. 75f. Dissertação (Mestrado em Proteção de Plantas) - Faculdade de Ciências Agronômicas, Botucatu, 2002.

TANADA, Y.; KAYA, H.K. Fungal Infections. In: .(Ed.). Insect pathology. San Diego: Academic

Press, 1992. p. 318-387. 
VAN DER GEEST, L.P.S.; ELLIOT, S.L.; BREEUWER, J.A.J.; BEERLING, E.A.M. Diseases of mites. Experimental and Applied Acarology, v.24, p.497-560, 2000.

WENZEL, I.M. Patogenicidade de Lecanicillium lecanii (Zimm.) Zare \& Gams ao ácaro rajado Tetranychus urticae (Acari: Tetranychidae) e sua compatibilidade a agrotóxicos e organismos biocontroladores utilizados na cultura do crisântemo. 2005. 84f. Tese (Doutorado em Agronomia - Proteção de Plantas) - Faculdade de Ciências Agronômicas, Botucatu, 2005.

WENZEL, I.M.; BATISTA FILHO, A.; ALMEIDA, A.M.B.; MINEIRO, J.L.C. Efeitos de produtos fitossanitários sobre o desenvolvimento e patogenicidade do fungo Lecanicillium lecanii. Arquivos do Instituto Biológico, São Paulo, v.71, p.172-174, 2004. Suplemento. Trabalho apresentado na REUNIÃO ANUAL DO INSTITUTO BIOLÓGICO, 17., 2004, São Paulo, SP. Resumo 131.

YEO, H.; PELL, J.K.; ALDERSON, P.G.; CLARK, S.J.; PYE, B.J. Laboratory evaluation of temperature effects on the germination and growth of entomopathogenic fungi on their pathogenicity to two aphids species. Pest Management Science, p.156-165, 2003.

Recebido em 24/7/09

Aceito em18/4/11 